\title{
Analysis of Verbal Interactions in Problem-based Learning
}

Sun $\mathrm{A} \mathrm{Oh}{ }^{1}$, Eun Kyung Chung ${ }^{2}$, Young Jong Woo' ${ }^{2}$ Eui Ryoung $\mathrm{Han}^{2}$ and Young Ok Kim ${ }^{3}$

${ }^{1}$ The Brain Korea 21 Project, Center for Biomedical Resources, Chonnam National University, Departments of ${ }^{2}$ Medical Education, and ${ }^{3}$ Pediatrics, Chonnam National University Medical School, Gwangju, Korea

\section{문제바탕학습 참여자의 언어적 상호작용 분석}

${ }^{1}$ 전남대학교 의생명인력사업단, 전남대학교 의과대학 ${ }^{2}$ 의학교육학교실, ${ }^{3}$ 소아청소년과학교실

오선아, 정은경 ${ }^{2}$, 우영종 ${ }^{2}$, 한의령 $^{2}$, 김영옥 $^{3}$

Purpose: Problem-based learning (PBL) is a constructive learning environment that solves ill-structured problems through collaborative learning. The purpose of this study was to analyze the interaction of students and a tutor in a small-group PBL discussion. This study examined how the types of interactions are composed over the meeting.

Methods: Fourteen third-year subjects from Chonnam National University Medical School, Korea formed two tutorial groups. Two tutorial sessions were videotaped and analyzed. All videotapes were transcribed to analyze the interaction type. The criteria of interaction analysis were learning-oriented interaction (exploratory questioning, cumulative reasoning, handling conflicts about the knowledge), procedural interactions, and irrelevant task interactions.

Results: Nearly all discourses between tutors and students were learning-oriented interactions. The results showed that students spent more time on cumulative reasoning. In contrast, tutors implemented more exploratory questioning. Little time was spent on handling conflicts about knowledge and procedural and irrelevant/off-task interactions.

Conclusion: To improve critical thinking and problem-solving competence in PBL, we should consider various efforts to encourage discussion about conflicting knowledge. A PBL tutor training program should be provided to facilitate PBL group discussions.

Key Words: Problem-based learning, Verbal interaction analysis

\section{서론}

협력학습(cooperative learning)은 동료 학생들 간의 상호 작용활동을 통해 학습과제를 해결하면서 학습내용을 비판적 으로 이해할 수 있고 지식을 정교화할 수 있다. 또한 협력학습 은 새로운 학습내용을 기존의 지식구조에 통합하려고 노력함
으로써 표층적인 학습보다는 심층적인 학습이 가능하며 자신 의 의견을 상대방에게 전달하면서 의사소통능력이 길러지는 장점이 있다[1,2]. 이러한 협력학습의 예가 문제바탕학습 (problem-based learning, PBL)이다. PBL은 비구조화된 문 제를 협동적 학습을 통해 해결하는 구성적 학습환경이다. 성 공적인 $\mathrm{PBL}$ 이 되기 위해서는 집단 구성원 간의 적극적인 상 호작용 활동이 중요하다. $\mathrm{PBL}$ 소집단 협력학습 과정을 활성
Received: March 22, 2010 - Revised: May 9, 2010 - Accepted: May 10, 2010 Corresponding Author: Eun Kyung Chung

Department of Medical Education, Chonnam National University Medical School, 5 Hak 1-dong Dong-gu, Gwangju 501-746, Korea

TEL) 062-220-4178, 4175 FAX) 062-236-3653 E-mail) ekcmedu@chonnam.ac.kr
Korean J Med Educ 2010 Jun; 22(2): 131-139. doi: 10.3946/kjme.2010.22.2.131.

pISSN: 2005-727X elSSN: 2005-7288

(C) The Korean Society of Medical Education. All rights reserved. 
화하기 위해서는 토의과정에서 학생들이 어떻게 의사소통을 하며 문제해결을 하는지에 관한 맥락중심적이고 상황중심적 인 연구가 필요하다.

그러나 지금까지의 PBL에 관한 연구는 주로 결과지향적인 양적 연구 중심으로 이루어졌다는 Visschers-Pleijers et al. [3]의 분석이 있다. 즉, $\mathrm{PBL}$ 과정이 끝난 후 설문작성을 통해 $\mathrm{PBL}$ 에 대한 전체적인 평가를 하는 것 $[4,5,6]$ 과 $\mathrm{PBL}$ 을 통해 자기주도적 학습능력, 비판적 사고, 자기효능감, 인식론적 신 념의 변화에 어떤 영향이 있는가와 이러한 변인과 학업성취 도와의 관련성을 평가한 것[7,8,9,10,11]이 있었다. 그리고 $\mathrm{PBL}$ 시행 후에 학생들의 수업만족도를 평가하는 연구[12]가 있다. 이러한 연구는 PBL이 종료된 후 또는 그 전후에 설문 조사를 통해 학생들의 반응을 조사한 것으로 PBL이라는 학 습과정 자체를 평가한 것은 아니다. PBL에 대한 질적 연구를 시도했던 Kim et al. [13]의 연구도 PBL 학습과정에 대한 인 식과 반응을 보기 위해 설문지와 개별 인터뷰를 실시하였으 나 PBL 상황에서 실제로 학습이 어떻게 이루어지고 있는가 를 보는 데는 한계가 있었다. PBL이 동료와의 의사소통을 통 해 학습자 스스로 자신의 지식을 구성해가는 협력학습이라고 고려할 때 $\mathrm{PBL}$ 의 평가도 학습과정에 초점을 둔 연구가 필요 하다.

따라서 이 연구의 목적은 PBL이라는 구성적 학습상황 속 에서 어떤 유형의 언어적 상호작용이 이루어지고 있는가를 분석한 것이다. $\mathrm{PBL}$ 과정에서 학생들은 얼마나 과제지향적 인 과정에 몰두하고 있으며 이러한 과정 속에서 나타나는 상 호작용 유형을 분석해 보고 학생과 튜터 간의 대화의 질을 평 가해보면 PBL이 학생들의 문제해결과 지식구성에 어떤 도움 이 되고 있는가를 살펴볼 수 있을 것이다.

\section{대상 및 방법}

\section{1. 연구대상 및 절차}

연구대상은 전남대학교 의학과 3학년으로 두 개의 PBL 소 집단 학생 14 명이다. 각 소집단은 학생 7 명과 1 명의 튜터로 구성되었다. $\mathrm{PBL}$ 은 2 회의 소집단 토론과 1 회의 전체토론 (colloquium)으로 이루어졌다. 첫 번째 소집단 토론에서는 $\mathrm{PBL}$ 사례에 대한 가설을 설정하고 증거와 추론에 근거한 해 결책을 모색하면서 학습과제(learning issues)를 도출한다. 첫 번째 소집단 토론이 끝난 후 학습자는 소집단 토론에서 도 출한 학습과제 해결을 위해 자기학습(self-study)을 한 다음 2차 소집단 토론에서 그 결과를 발표해야 한다. 두 번째 소집 단 토론이 끝나면 PBL 모듈을 개발한 교수와 함께 하는 전체 토론이 있다. 이 연구에서는 첫 번째 소집단 토론과정의 학생 과 튜터의 언어적 상호작용 과정을 분석했다. 연구에 앞서 학 생들에게 연구목적과 방법을 설명하고 연구를 위해 비디오 촬영과 대화분석에 참여해 줄 것을 권유했고 동의를 받았다.

두 팀의 첫 번째 소집단 토론 전 과정을 비디오로 찍었는데 전체 녹화시간은 약 4시간 정도이다. 비디오로 녹화된 전체토 론 내용을 대화 유형별로 분석하기 위해 모두 한글로 전사 (transcript)했다. 토의주제는 두 팀 모두 "미숙아의 폐질환" 이었고 학습목표는 '저출생 체중아 및 미숙아를 정의하고 미 숙아가 정상아에 비하여 불리한 점을 열거한다, '신생아 호흡 곤란증후군의 주요 발생기전과 처치에 대하여 설명한다, '신 생아 호흡곤란증후군의 합병증을 설명한다이다. 각 팀의 토 의시간은 약 2 시간 정도였다.

\section{2. 대화 분석방법}

\section{1) 분석단위}

$\mathrm{PBL}$ 소집단 토의과정을 비디오로 녹화한 다음 그 내용 을 문자로 다 풀어 쓴 다음, 유의미한 분석단위인 발화 (utterance)로 분석했다. 그 후에 발화를 범주대로 코딩하고 그 코딩 과정은 두 연구자의 합의 절차를 통해 이루어졌다. 발 화는 개별적인 메시지 단위로 한 사람에 의해 표현된 것이어 야 하고 하나의 주제를 담은 의사소통적인 기능이 있어야 한 다. 즉, 질문, 평가, 논쟁과 같이 자신의 생각을 포함한 주제를 담고 있어야 한다. 그러므로 발화는 단 한마디의 단어부터 몇 줄의 문장에 이를 수 있다.

\section{2) 코딩틀(coding scheme)}

전사된 모든 내용은 Visschers-Pleijers et al. [3]의 코딩틀 에 근거해서 분석되었다. PBL 튜토리얼 과정에 일어난 모든 대화내용을 학습지향적인 상호작용(learning-oriented interactions), 절차적 상호작용(procedural interactions), 과 
Table 1. Coding Scheme (From Visschers-Pleijers, et al. Med Educ 2006; 40: 129-137, with permission from John Wiley and Sons) [3]

\begin{tabular}{|c|c|}
\hline Category & Definition \\
\hline I. Learning-oriented interactions & Utterance reflecting on task activities. \\
\hline 1. Exploratory questioning & $\begin{array}{l}\text { Group members engage critically but constructively with each other's ideas by asking high-order } \\
\text { questions or considering alternative explanations. }\end{array}$ \\
\hline Open question & - Simple questions that ask for new information and fact. \\
\hline Critical question & - Checking the question of another person's utterance. \\
\hline Verification question & - Questions in which one's own ideas or reasoning are checked. \\
\hline Alternative argument & - A logical extension of a previous utterance reflecting alternative reasoning \\
\hline 2. Cumulative reasoning & $\begin{array}{l}\text { Group members builds positively but uncritically on what is said by a group member; this } \\
\text { leads to an automatic consensus and group members construct a 'common knowledge' by } \\
\text { accumulation. }\end{array}$ \\
\hline Statement & $\begin{array}{l}\text { - An utterance in which factual information is provided without reflecting reasoning and just } \\
\text { read a material and note. }\end{array}$ \\
\hline Other argument & - A logical extension of a previous utterance reflecting reasoning, explanation. \\
\hline Other question & $\begin{array}{l}\text { - A question asking for a choice between } 2 \text { or more options or a request for evaluation eliciting } \\
\text { a short answer. }\end{array}$ \\
\hline $\begin{array}{l}\text { Judgement acceptance } \\
\text { /Confirmation }\end{array}$ & - A confirmation or acceptance or a previous content-related utterance. \\
\hline $\begin{array}{l}\text { 3. Handling conflicts about } \\
\text { knowledge }\end{array}$ & $\begin{array}{l}\text { Group members acknowledge and discuss contradictory information, characterized by } \\
\text { expressing disagreement, negation of previous utterances or counter arguments. }\end{array}$ \\
\hline Counter argument & $\begin{array}{l}\text { - A logical extension of a previous utterance reflecting reasoning that contradicts the previous } \\
\text { utterance }\end{array}$ \\
\hline $\begin{array}{l}\text { Judgement negation } \\
\text { /Disagreement }\end{array}$ & - Negation of a previous content-related utterance or a negative answer to a question. \\
\hline Evaluation & $\begin{array}{l}\text { - Content-related personal opinion or judgement with regard to your own or someone else's } \\
\text { knowledge and understanding of the problem. }\end{array}$ \\
\hline II. Procedural interaction & $\begin{array}{l}\text { Utterance related to the collaboration process that focus on handling organizing or executing } \\
\text { the problem. }\end{array}$ \\
\hline III. Irrelevant/Off-task & Utterance not related to the task, a period of silence. \\
\hline
\end{tabular}

제해결과 무관한 상호작용(irrelevant/off-task)로 나누었다. 학습지향적인 상호작용은 탐색적 질문(exploratory questioning), 누적적 추론(cumulative reasoning), 지식에 대한 갈등 해결(handling conflicts about knowledge)로 분류된 다. 이에 대한 설명은 Table 1과 같다.

탐색적 질문은 구성원들이 고차원적인 질문이나 대안적인 설명을 하여 서로 다른 사람의 아이디어에 비판적 · 구성적으 로 참여하는 것을 의미한다. 탐색적 질문의 하위영역에는 '개 방형질문(open questions)'이 있는데 이는 새로운 정보에 대 해 그 정보의 특질, 의미, 예, 차이, 유사성, 이유 등을 자유롭 게 물어보는 질문이다. 즉, 개방형 질문은 화자의 비판적 - 논
리적인 의견이 아닌 있는 그대로의 사실을 확인하는 것이다. '비판적 질문(critical questions)'은 다른 사람의 의견을 점검 하거나 이를 확인하는 것을 말하며 '확증적 질문(verification questions)'은 자기 자신의 아이디어에 의문과 추론을 점검하 는 것이다. 즉, 자신의 생각이 맞는지 아닌지를 동료에게 물어 보고 확인하는 질문을 말한다. '대안적인 논증(alternative argument' '은 이미 주어진 설명에 대한 대안적인 설명을 하 면서 추론을 반영하여 이전 발언의 논리를 확장시키는 것을 말한다.

누적적 추론이란 $\mathrm{PBL}$ 에 참여하는 구성원들이 어떤 동료가 말한 내용에 대해서 긍정적이고 무비판적으로 받아들이는 대 
화 내용이다. 즉, 다른 동료의 의견에 대한 수용은 자동적인 합의를 이끌어 내고 이렇게 구성원들이 내놓은 다양한 내용 이 자연스럽게 쌍임으로써 '공통 지식(common knowledge)'을 구성하고 이를 통해 문제해결의 단서를 찾게 되는 것을 말한다. 이러한 누적적 추론에는 '진술(statement)'와 ‘다 른 논증(other argument)', '다른 질문(other question)' 그리 고 '결정수용/확인(judgment acceptance/confirmation)'이 있다. '진술'은 사실적인 정보가 제공되는 발화로 이것은 추론 을 반영하지 않고 학생들이 주어진 책이나 자료의 정보를 있 는 그대로 읽는 것을 말한다. '다른 논증'이란 추론을 반영하 여 이유, 조건, 결론 등을 반영하여 이전 발언의 논리를 확장 하는 것을 말한다. '다른 질문은 둘 혹은 그 이상의 옵션 중에 서 선택을 요구하는 질문으로 짧은 대답을 하게 해 평가하는 질문을 말한다. '결정수용/확인'은 이미 나온 어떤 대화 내용 과 관련된 내용의 승인과 수용을 말한다.

지식에 대한 갈등해결 상호작용이란 구성원들끼리 어떤 내용에 대해 의견이 상충되거나 반대되는 논쟁을 하며 문 제를 해결하는 것을 말한다. 그 하위영역으로는 '반대 논쟁 (counter argument)', '판단 부정/불일치(judgment negation/disagreement)', '평가(evaluation)'가 있다. '반대논쟁'은 상대방이 내놓은 발언과 상충되는 추론을 반영하여 기존 발 언의 논리적 확장을 하는 것을 말하며, '판단 부정/불일치는 내용과 관련된 발언의 부정이나 부정적인 답변을 의미한다. '평가'는 자신이나 다른 사람의 지식과 문제이해에 대한 내용 관련 개인적인 의견이나 판단을 말한다.

절차적 상호작용은 문제를 처리하고 조직화하고 실행하는 데 초점을 둔 협동과정과 관련된 발언으로 역할분담이나 의 견 발표순서 그리고 토론내용의 순서 지정이나 의견 정리방 법에 관한 것이다. 과제해결과 무관한 상호작용은 주어진 문 제해결과 관련 없는 것으로 토의과정의 문제점이나 컴퓨터 문제, 모듈 구성의 문제점 지적 그리고 토론에 참여하지 않고 컴퓨터 문제 등을 언급하면서 탈맥락적인 혼잣말을 하는 것 을 말한다.

\section{3) 채점자 신뢰도}

두 명의 채점자가 한 팀씩 맡아 대화내용을 읽고 코딩틀에 따라 대화내용을 분류했다. 분석하는 과정에서 발생한 해석 상의 모호한 내용은 두 채점자가 같이 읽고 그 대화맥락에서
Table 2. The Types of Students' Dialogue Analysis during PBL Tutorials

\begin{tabular}{cc}
\hline \multicolumn{1}{c}{ Category } & $\mathrm{n}(\%)$ \\
\hline I. Learning-oriented interactions & $601(83.8)$ \\
1. Exploratory questioning & $181(25.2)$ \\
Open question & $109(15.2)$ \\
Critical question & $21(2.9)$ \\
Verification question & $11(1.5)$ \\
Alternative argument & $40(5.8)$ \\
2. Cumulative reasoning & 342 (47.7) \\
Statement & $166(23.2)$ \\
Other argument & $113(15.7)$ \\
Other question & $30(4.2)$ \\
Judgement acceptance/Confirmation & $33(4.6)$ \\
3. Handling conflicts about knowledge & $78(10.9)$ \\
Counter argument & $15(2.1)$ \\
Judgement negation/Disagreement & 28 (3.9) \\
Evaluation & $35(4.9)$ \\
II. Procedural interaction & $84(11.7)$ \\
III. Irrelevant/Off-task & $32(4.46)$ \\
& $717(100.0)$ \\
\hline
\end{tabular}

PBL: problem-based learning.

의미하는 바를 토의한 후 발화의 특징을 분석했다. 두 채점자 간 내적 일치도 분석을 위해 30 분 분량의 대화내용을 각각 분 석한 후 채점자 간 일치도를 산정했는데 0.87 이었다(Cohen's kappa).

\section{결과}

Table 2와 Table 3은 PBL 튜토리얼에서의 학생과 튜터의 대화분석 결과이다. 위 결과를 보면 PBL 튜토리얼에서 학생 과 튜터 모두 학습지향적인 활동에 집중하고 있음을 보여준 다. 즉, 학생들은 주어진 과제를 해결하고자 하는 학습지향적 인 활동인 탐색적 질문, 누적적 추론, 지식에 대한 갈등해결의 대화에 $83.8 \%$, 튜터는 $66.7 \%$ 를 하는 것으로 나타났다. 그리 고 절차적 상호작용은 학생과 튜터가 각각 $11.7 \%, 19.3 \%$, 과 제와 무관한 상호작용은 학생이 $4.46 \%$, 튜터가 $14.4 \%$ 로 나타 났다. 
$\overline{\text { Table 3. The Types of Tutor' Dialogue Analysis during PBL Tutorials }}$

\begin{tabular}{cc}
\hline \multicolumn{1}{c}{ Category } & $\mathrm{n}(\%)$ \\
\hline I. Learning-oriented interactions & $38(66.7)$ \\
1. Exploratory questioning & $17(29.8)$ \\
Open question & $10(17.5)$ \\
Critical question & $1(1.8)$ \\
Verification question & $1(1.8)$ \\
Alternative argument & $5(8.8)$ \\
2. Cumulative reasoning & $13(22.8)$ \\
Statement & $10(17.5)$ \\
Other argument & $3(5.3)$ \\
Other question & 0 \\
Judgement acceptance/ & 0 \\
Confirmation & $8(14.0)$ \\
3. Handling conflicts about knowledge & $1(1.8)$ \\
Counter argument & $1(1.8)$ \\
Judgement negation/ & \\
Disagreement & $6(10.5)$ \\
Evaluation & $11(19.3)$ \\
II. Procedural interaction & $8(14.4)$ \\
III. Irrelevant/Off-task & $57(100.0)$ \\
\hline Total &
\end{tabular}

PBL: problem-based learning.

그러나, 학습지향적인 상호작용에서도 학생과 튜터의 대화 유형에는 차이가 있었다. 학생은 누적적 추론 상호작용을 가 장 많이 한 반면, 튜터는 탐색적 질문 상호작용이 가장 많았 다. 보다 구체적인 대화분석의 내용은 다음과 같다.

\section{1. 학생의 대화분석}

\section{1) 학습지향적인 대화}

a. 탐색적 질문

탐색적 질문은 전체 대화의 $25.2 \%$ 로 나타났는데 그 하위영 역별로 살펴보면 다음과 같다. 개방적 질문이 $15.2 \%$, 비판적 질문이 $2.9 \%$, 확증적 질문이 $1.5 \%$, 그리고 대안적 논증이 $5.8 \%$ 로 분석되었다. 개방형 질문은 새로운 정보에 대해 화자 가 비판적 논리적 의견을 가지고 물어보기보다는 있는 사실 의 특징이나 의미, 이유, 차이 등을 물어보는 질문이다. 학생 들은 주로 모듈자료에 나와 있는 내용을 사실적으로 다음과 같이 질문했다.
S5: “청색증 소견을 보이므로 심장질환에 기인한 것이죠?" S1: "그런데 양수가 탁한 노란색인데 이럴 때 나타날 수 있 는 태아문제는 무엇일까요?"

비판적 질문은 다른 사람의 의견을 점검하거나 이를 확인 하는 대화이다. 이처럼 비판적 질문은 다른 사람이 내놓은 의 견에 대해서 자신의 의견을 덧붙여 그 의견의 진위 여부를 점 검하고 확인하는 질문형태로 다음과 같은 발화이다.

S3: “그런데...어... 탁한 노란색 양수라는 것은 정상양수 소 견은 아니지요?"

S7: “연령을 그렇게 교정연령으로만 계산하지 말고 역연령 으로 계산해 봐야지 않을까요?"

확증적 질문은 자기 자신의 아이디어에 의문과 추론을 점 검하는 것으로 자신의 생각이 맞는지 아닌지를 동료에게 물 어보고 확인하는 질문을 말한다. 다음의 대화는 확증적 질문 의 하나이다.

$\mathrm{S} 2:$ "제 생각에는 양수 과소증의 원인으로 신형성 저하증 및 요관폐쇄 등도 고려할 수 있는데 환아 입원 시 혈청 에서 시행한 신기능검사결과 bun $13.9 \mathrm{mg} / \mathrm{dL}, \mathrm{cr} 1.2$ $\mathrm{mg} / \mathrm{dL}$ 로 연령에 비해 creatinine이 다소 높은 것 같아 요. 그래서 이 아이는 신장기능에 좀 문제가 있는 것 같은데요. 신장초음파와 같은 신요로계 검사를 해보면 어떨까요?"

대안적인 논증은 이미 주어진 설명에 대한 대안적인 설명 을 하면서 추론을 반영하여 이전 발언의 논리를 확장시키는 것을 말한다. 계속적으로 논의되어 온 내용과는 좀 더 다른 방 향의 논리를 확장하는 것으로 다음과 같은 대화내용을 말한 다.

$\mathrm{S} 2:$ 생후 40일째 하루 5 6차례 무호흡 및 산소포화도 감 소가 자주 보이는데, 이와 같은 문제가 신생아 감염에 만 연관되었다고 보기는 어렵지 않나요? 이 아이는 출 생 시 신생아 호흡곤란 증후군으로 인공환기요법 및 
인공폐표면 활성제 보충요법을 받았고 동맥관개존이 있어서 수술도 했잖아요."

S1: “... 그러므로 제 생각엔 지금 이 산모의 질병에 초점이 있는 것이 아니라 신생아의 질병이 아닌가 싶어요."

$\mathrm{PBL}$ 협력학습 동안 학생들이 했던 탐색적 질문의 대부분 은 모듈 학습자료에 나온 내용에 대해 정의하고 확인하는 개 방형 질문이었다. 다른 사람과 자기 자신의 아이디어를 점검 하고 확인하는 비판적이고 확증적인 질문의 빈도는 상대적으 로 낮게 나타났다. 그리고 대안적인 논증이 단순한 개방형 질 문에 이어 많은 질문 유형으로 나타났는데 실제로 그 대화의 질을 보면 한 학생이 내놓은 의견에 대해 다른 학생이 그 의견 을 확장하고 수용하는 상호작용적인 것이라기보다는 각각의 학생이 산발적으로 각자의 새로운 대안적인 아이디어를 제시 하고 있었다. 즉, 서로 각자의 아이디어를 내놓기는 하지만 이 아이디어가 문제를 해결하기 위해 어떻게 활용될 수 있고 가 능한가에 대한 학생들 상호 간에 발전된 논의는 별로 없었고 단지 의견만 이 사람 저 사람 제시하는 수준이 대부분이었다.

b. 누적적 추론

누적적 추론에서 학생들의 대화는 진술(23.2\%)과 다른 논 증(15.7\%)이 대부분을 차지하고 있다. 이에 반해 다른 질문 (4.2\%)이나 결정수용/확인(4.6\%)에 관한 대화는 상대적으로 적게 나타났다.

진술은 학생들이 주어진 모듈자료에 있는 정보를 사실대로 전달하는 것으로 주로 모듈 자료가 제공된 직후에 많이 나타 났다. 다음과 같은 진술은 학생들이 주어진 $\mathrm{PBL}$ 모듈 자료를 보고 사실/문제(fact/problem)를 분석하면서 한 것이다.

S3: "재태주수 27 주 3 일의 태아이고 출생 시 체중은 $650 \mathrm{~g}$ 입니다."

S4: "양수가 끈적거리면서 탁한 노란색이고 손발입술에 청 색증이 보입니다."

다른 논증이란 추론을 반영하여 주어진 자료나 다른 학생 의 발화에 이유, 조건, 결론 등을 근거로 자신의 의견을 확장 하는 것이다. 즉, 한 학생이 한 발화에 대해 자신의 의견을 추 가한 것으로 다음과 같은 대화내용이다.
S2: "조산이고 미숙아여서 아직 폐가 성숙하지 못했거나 발 달되지 못했으리라 생각됩니다."

S4: “청색증 같은 경우에는 일반적으로 심장질환에 이상이 있어서 오는 것이 있지만 산모가 심한 양수과소증 소견 을 보였으므로 양수과소증에서 동반되는 폐형성 저하 또한 고려되어야 할 것 같아요."

다른 질문은 모듈에서 제시된 사실과 문제를 가지고 어떤 질환인지 결정하는 데 있어 상대방에게 가능한 답을 결정하 도록 요구하는 것으로 주로 토론 후반부에 많이 이루어졌다.

S1: “음... 그럼... 이와 같은 조산을 일으킨 원인이 태아 이 상, 태반 이상, 산모의 원인 중 어디에 속하는 문제였 을까요?"

$\mathrm{S7}$ : “청색증 진단을 위해 CT 촬영이 필요한가요?"

S3: “산모는 과거력 소견이 없는 23세 초산부이므로 조산 의 원인으로 산모의 원인보다는 태아의 이상에 초점을 두고 태아문제에 속하는 각각의 개별원인을 고려하는 것은 어떤가요?"

결정수용/확인은 이미 나온 어떤 대화 내용과 관련된 내용 의 승인과 수용을 말하는 것으로 다른 사람의 의견에 대해 긍 정하고 받아들이는 것을 말한다.

S1: "이 신생아가 출생 후 이와 같은 응급상태에 빠진 것 은... 음... 초극소 저체중 출생 미숙아이므로 신생아 호 흡 곤란 증후군을 비롯한 심장 호흡기계 불리한 점을 가지고 있기 때문이라는 거 다들 동의하시는 거죠?" (대다수의 학생들): "네!"

누적적 추론에서 학생들이 가장 많이 한 상호작용은 주어 진 자료의 내용을 자신의 언어가 아닌 있는 그대로 전달하는 단순한 진술이었고 그 다음은 다른 동료학생이 한 말에 대해 여러 가지 이유나 결과, 논거를 들어 더 확대된 논리로 이끄는 다른 논증이 많았다. 반면에 내용에 대한 선택을 요구하는 다 른 질문, 주어진 내용에 대한 승인과 수용에 대한 상호작용은 
상대적으로 적게 나타났다. 이러한 발화는 주로 토의가 어느 정도 마무리되는 토의 후반부에 나타났다. 그러나, 토의 과정 에서 행해진 다양한 발화가 지속적이고 상호작용적인 대화를 통해 이루어지기보다는 학생 개개인이 혼잣말처럼 던지는 경 우가 많았고 다른 학생의 의견에 대한 적절한 질문과 확인을 하고 도출된 결론에 대해 합의적 수용을 하는 발화는 드물었 다.

c. 지식에 대한 갈등해결

지식에 대한 갈등해결은 $10.9 \%$ 로 나타났는데 반대 논쟁이 $2.1 \%$, 판단 부정/불일치가 $3.9 \%$, 평가가 $4.9 \%$ 로 나타났다. 반대논쟁은 상대방이 내놓은 발언과 상충되는 추론을 반영하 여 기존 발언의 논리적 확장을 하는 것을 말한다. 판단 부정/ 불일치는 내용과 관련된 발언의 부정이나 부정적인 답변을 의미한다. 평가는 자신이나 다른 사람의 지식과 문제 이해에 대한 내용관련 개인적인 의견이나 판단을 말한다.

S1: "1부의 chief complain이 미숙아일 때 나오는 현상인 것 같습니다(evaluation). 우리가 그동안 태아에 집중 했는데 산모에게도 문제가 있다는 걸 생각해 봅시다 (alternative argument).”

S5: “지금 산모측 문제를 고려하자고 하셨지만 산모력을 조 사한 2부 내용을 고려하면 산모는 조산을 일으키는 원 인을 가지고 있지 않다고 볼 수 있어요(judge negation)."

S2: “그런데... 그보다 여기서 더 중요한 것은 미숙아에서 나타날 수 있는 불리한 문제점과 산모원인보다는, 제 생각에는 태반이상 및 산모 자궁이상과 같은 태아가 자 라는 자궁 내 환경문제가 고려되어야 할 것 같아요 (counter argument)."

학생들은 PBL 토의과정 동안 주로 다른 사람의 의견을 듣 고 제시된 자료에 대한 개방형 질문을 통해 계속적으로 많은 지식을 무비판적으로 누적적으로 쌓는다. 그리고 거의 모든 모듈 자료가 제시되는 시점에서 모듈에서 제공하는 진단명이 나 질환을 확증할 때에 구성원 간에 이견과 갈등 양상을 보이 는 것으로 나타났다.

\section{2) 절차적 상호작용}

절차적 상호작용은 $11.7 \%$ 로 분석되었다. 절차적 상호작용 은 주로 토론절차와 토론할 내용 순서와 같은 것으로 다음과 같은 대화이다.

$\mathrm{S1}:$ "3부 자료를 김 $\bigcirc$ 선생님이 읽어주세요."

$\mathrm{S} 1$ : "먼저 여기서 나온 fact를 살펴보면서 그러한 현상이 일어날 원인을 분류해 보면 좋겠습니다."

\section{3) 과제와 무관한 상호작용}

과제와 무관한 상호작용은 4.5\%로 나타났다. 과제와 무관 한 상호적용은 모듈의 부적절성을 지적한다든가 서기가 정리 하는 데 있어 컴퓨터 프로그램 수행과 관련된 문제점을 지적 하거나 내용과 무관하게 지엽적으로 이루어지는 산발적인 대 화가 포함되었다.

$\mathrm{S} 1$ : (서기에게) "창 크기 사이즈를 줄여봐."

$\mathrm{S} 7:$ (서기를 보면서) "더 줄여도 될 것 같은데."

$\mathrm{S} 2$ : (혼잣말로) "오늘 모듈내용이 이상해!"

\section{2. 튜터의 대화분석}

튜터의 대화는 학생에 비해 적게 나타났다. 이는 PBL 시작 전 튜터 모임에서 튜터의 역할을 지식을 전달하고 전수하는 지시적인 역할이 아닌 조력자, 촉진자, 안내자로서의 역할 [14]로 상기시켰기 때문이라고 추론할 수 있다. 튜터의 대화 를 보면 학생들과는 달리 누적적 추론보다는 탐색적 질문이 더 많은 것으로 나타났는데 이는 토의 주제에 대해 튜터가 학 생보다 사전지식이 많고 토론의 활성화를 위해서 적정한 시 점에서 주의를 환기하는 질문을 해주고 있었다고 할 수 있다.

튜터는 주로 진행과 관련된 발언을 많이 했고 모듈자료를 배분한 다음 침묵 속에 있다가 학생들의 토론이 초점에서 벗 어날 때 자신의 의견을 제시하기도 하고 모듈에서 나타난 상 황을 설명하기도 했다. 가끔씩은 장황한 부적절한 설명을 하 기도 했고 튜터의 발언에 따라 학생들의 토의내용과 토의방 향이 상당부분 영향받고 있음이 관찰되었다. 


\section{고찰}

$\mathrm{PBL}$ 소집단 토의 과정에서 이루어지는 학생들의 대화내용 은 주로 과제해결을 위한 탐색적 질문, 누적적 추론, 지식에 대한 갈등해결 과정과 같은 학습지향적인 활동이 대부분인 것으로 나타났다. 절차적 상호작용과 과제와 무관한 상호작 용은 상대적으로 적게 나타났다. 이 결과는 De Grave et al. [15]과 Visschers-Pleijers et al. [3]의 결과와 유사한데 이것 은 PBL 진행에 대해 튜터와 학생들의 오리엔테이션이 잘 이 루어진 결과라고 해석할 수 있다.

$\mathrm{PBL}$ 튜토리얼에서 일어나는 학생과 튜터의 대화 대부분이 학습지향적인 것이었는데 실제로 그 대화의 구성을 보면 다 음과 같다. 학생은 학습지향적인 상호작용에서도 누적적 추 론이 $47.7 \%$ 로 가장 많은 비율을 차지한 반면 튜터는 탐색적 질문을 $29.8 \%$ 로 많이 하고 있었다. 학생은 문제를 해결해 가 기 위해 해당 모듈 자료의 정보에 대한 사실적 진술이 많았으 며 이 자료에서 나온 내용을 근거로 자신의 의견을 조금씩 전 달하고 덧붙여가면서 다른 논증으로 발전시켜 갔다. 이에 반 해 튜터는 학생들에게 개방적 질문이나 대안적인 논증을 제 시해 초점을 벗어난 토론을 이끄는 대화를 누적적 추론보다 더 많이 사용하는 것으로 추론할 수 있다.

이 연구의 시사점은 PBL 튜토리얼 학습과정을 직접 들여 다보고 그 속에서 문제해결을 위해 어떤 대화가 어느 정도 이 루어지고 있는가를 분석했다는 데 있다. $\mathrm{PBL}$ 에 관한 많은 연 구가 $\mathrm{PBL}$ 효과를 평가해 왔지만 실제 $\mathrm{PBL}$ 과정이 어떻게 구 성되고 학생들과 튜터는 어떤 대화를 통해 문제를 해결하고 있는지 알 수는 없었다. 이 연구결과를 보면 문제해결을 위해 학생들은 대부분 학습지향적인 대화를 주로 많이 나누지만 그 대화의 내용은 비판과 논쟁적인 대화보다는 주로 누적적 인 추론이 많았고 주어진 학습모듈 자료에 따라 여러 학생들 의 진술과 다른 논쟁 그리고 개방적 질문과 같은 피상적인 대 화가 많이 이루어졌음을 알 수 있다. 주제에 대한 보다 논쟁적 이고 이러한 갈등적인 논쟁을 조정하고 해결하는 반론과 확 증적인 질문과 논의는 상대적으로 적게 드러났다. 그동안 여 러 연구를 통해서 PBL이 의사소통능력, 비판적 사고능력, 메 타인지 능력을 증진시켜 준다는 연구[15,16,17]가 있었는데
PBL이 이러한 능력을 보다 향상시킬 수 있으려면, PBL 모듈 내용에 보다 논쟁적이고 비판적인 사고를 할 수 있는 주제가 많이 포함되도록 해야 할 것이다. 또한 튜터가 학습과정에서 소극적인 구경꾼이 아닌 질문, 탐색, 반성적 성찰, 제안, 도전 과 같은 집단 촉진 기술을 가질 수 있도록 해야 하고 이에 대 한 튜터 훈련 프로그램이 필요하다고 할 수 있다.

향후 $\mathrm{PBL}$ 튜토리얼의 대화분석의 연구를 위한 제언은 다 음과 같다.

첫째, 모듈 주제와 교육환경에 따라 대화유형의 차이가 있 는가를 살펴볼 필요가 있을 것이다. 모듈의 내용이 논쟁적이 고 비판적인 판단력을 요구하는 것이라면 대화유형도 지식에 대한 갈등해결과 탐색적 질문이 늘어날 것이다.

둘째, 튜터의 유형에 따른 PBL 토론의 질적 차이를 살펴볼 필요가 있다. 이 연구에서는 2 명의 $\mathrm{PBL}$ 튜터가 참여하여 소 집단 토론을 이끌었는데 적은 빈도의 튜터 발화가 있었지만 튜터의 발화태도가 어떠한가에 따라 이후 대화의 질과 빈도 에 많은 영향을 미치고 있음을 볼 수 있었다. 향후 연구에서는 튜터의 교수스타일[18]에 따라 학생들의 상호작용 유형이 달 라지는지 살펴보고자 한다.

셋째, 튜토리얼 과정에서 일어나는 대화의 질과 학습자가 도출한 학습과제(learning issue)간의 관계를 분석할 필요가 있겠다. 현재 분석된 두 팀에서도 팀의 토의 내용과 분위기에 따라 같은 모듈이라도 도출된 학습과제에 있어서 차이가 있 었다. 이는 $\mathrm{PBL}$ 튜토리얼에서 이루어지는 상호작용의 양상 에 따라 그 모듈에서 요구하는 학습목표의 달성여부에 영향 을 미칠 수 있음을 보여주는 것이다. 그러므로 앞으로의 연구 에서는 보다 많은 소집단 튜토리얼 집단을 대상으로 상호작 용 과정과 학습과제 도출의 관계를 탐색하고자 한다.

\section{ACKNOWLEDGEMENTS}

This work was supported by the Research Fund from the Korean Society of Medical Education (2008). 


\section{REFERENCES}

1. Boxtel CV, Linden JV, Kanselaar G. Collaborative learning tasks and the elaboration of conceptual knowledge. Learn Instr 2000; 10: 311-330.

2. Gillies RM. The effects of cooperative learning on junior high school students during small group learning. Learn Instr 2004; 14: 197-213.

3. Visschers-Pleijers AJ, Dolmans DH, de Leng BA, Wolfhagen IH, van der Vleuten CP. Analysis of verbal interactions in tutorial groups: a process study. Med Educ 2006; 40: 129-137.

4. Lee JT, Choi JS, Kim SH, Paik NW. The experience and evaluation of problem-based learning in Inje University College of Medicine. Korean J Med Educ 1998; 10: 351362.

5. Chang BH, Lee YC, Kim BW, Kang DS, Kwak YS, Kang E, Seo KS, Kim IK, Lee JM, Jeong SH, Kim JY, Kim IS, Kim HI. The Implementation of problem-based learning in Kyungpook National University School of Medicine and its evaluation. Korean J Med Educ 2001; 13: 91-105.

6. Chung IW, Shin CJ, Han HS, Song YJ. The practice and evaluation of problem-based learning in College of Medicine, Chungbuk National University. Korean J Med Educ 1999; 11: 285-295.

7. Kim YM, Park YS, Chung CW, Kim MJ. Application of problem-based learning (PBL) for students' practice in maternity nursing. Korean J Women Health Nurs 2006; 12: 326-337.

8. Chae SJ, Shin JS, Eun HC, Lee YS. A study on the use of reflective journals in problem-based learning. Korean J Med Educ 2002; 14: 157-164.

9. Kim JY, Choi EY. Participation and academic achieve ment by learning styles in problem-based learning. J Korean Acad Fundam Nurs 2009; 16: 200-206.

10. Yoo MR, Choi YJ, Kang MS. The effects of PBL (problem-based learning) on the self-directed learning, critical thinking dispositions, and problem-solving process of nursing students. J Korean Acad Fundam Nurs 2009; 16: 46-55.

11. Rhee JA, Chung EK, Oh SA, Baik YH, Woo YJ. Changes of epistemological beliefs and self-regulated learning in problem-based learning. Korean J Med Educ 2009; 21: 153-161.

12. So YH, Lee YM, Ahn DS. An student's evaluation of the implementation of problem-based learning. Korean J Med Educ 2005; 17: 49-58.

13. Kim JH, Kim JY, Son HJ, Choi YH, Hong KP, Ahn BH, Uhm DY, Chin YE, Seo JD. A qualitative evaluation of problem-based learning curriculum by students' perceptions. Korean J Med Educ 2004; 16: 179-193.

14. Das M, Mpofu DJ, Hasan MY, Hasan TS. Student perceptions of tutor skills in problem-based learning tutorials. Med Educ 2002; 36: 272-278.

15. De Grave WS, Boshuizen HP, Schmidt HG. Problembased learning: cognitive and metacognitive processes during problem analysis. Instr Sci 1996; 24: 321-341.

16. Sungur S, Tekkaya C, Geban Ö. Improving achievement through problem-based learning. J Biol Educ 2006; 40: 155-160.

17. Gordon PR, Rogers AM, Comfort M, Gavula N, McGee BP. A taste of problem-based learning increases achievement of urban minority middle-school students. Educ Horiz 2001; 79: 171-175.

18. Leung KK, Lue BH, Lee MB. Development of a teaching style inventory for tutor evaluation in problem-based learning. Med Educ 2003; 37: 410-416. 